\title{
Dual-Gene, Dual-Cell Type Therapy against an Excitotoxic Insult by Bolstering Neuroenergetics
}

\author{
Tonya M. Bliss, ${ }^{1,2}$ Miranda Ip, ${ }^{1}$ Elise Cheng, ${ }^{1}$ Masabumi Minami, ${ }^{3}$ Luc Pellerin, ${ }^{4}$ Pierre Magistretti, ${ }^{4}$ and \\ Robert M. Sapolsky ${ }^{1}$ \\ Departments of ${ }^{1}$ Biological Sciences and ${ }^{2}$ Neurosurgery, Stanford University, Stanford, California 94305, ${ }^{3}$ Department of Molecular Pharmacology, \\ Graduate School of Pharmaceutical Sciences, Kyoto University, Kyoto 606-8501, Japan, and ${ }^{4}$ Institut de Physiologie, 1005 Lausanne, Switzerland
}

\begin{abstract}
Increasing evidence suggests that glutamate activates the generation of lactate from glucose in astrocytes; this lactate is shuttled to neurons that use it as a preferential energy source. We explore this multicellular "lactate shuttle" with a novel dual-cell, dual-gene therapy approach and determine the neuroprotective potential of enhancing this shuttle. Viral vector-driven overexpression of a glucose transporter in glia enhanced glucose uptake, lactate efflux, and the glial capacity to protect neurons from excitotoxicity. In parallel, overexpression of a lactate transporter in neurons enhanced lactate uptake and neuronal resistance to excitotoxicity. Finally, overexpression of both transgenes in the respective cell types provided more protection than either therapy alone, demonstrating that a dual-cell, dual-gene therapy approach gives greater neuroprotection than the conventional single-cell, single-gene strategy.
\end{abstract}

Key words: lactate; glucose; gene therapy; neuron death; energy; neurotoxicity

\section{Introduction}

There have been tremendous advances in our understanding of the events that mediate neuron death after excitotoxic neurological insults, such as hypoxia-ischemia or seizure. With this insight has come increasing potential for intervention, including gene therapy with neuroprotective genes. Overexpression of more than 20 different genes has been reported to decrease insultinduced neurotoxicity both in vitro and in vivo (for review, see Sapolsky, 2003). An early gene therapy approach was to overexpress the GLUT1 glucose transporter, on the basis of the notion that neurons rely primarily on glucose for energy, and that boosting their ability to use glucose would allow them to meet the energetic demands involved in containing the consequences of excitotoxic insults. In primary cultures, GLUT1 overexpression enhanced glucose uptake, spared energy metabolism and ATP levels during an insult, and enhanced the ability of neurons to remove glutamate from the synapse and calcium from the cytoplasm. Moreover, such overexpression decreased neurotoxicity both in vitro and in vivo in response to various excitotoxic insults and spared the functional integrity of neurons (Ho et al., 1995a; Lawrence et al., 1995; McLaughlin et al., 2000; Gupta et al., 2001).

The classical picture of neuronal preference for glucose has been challenged in recent years (for review, see Pellerin and Magistretti, 2003). An increasing body of evidence suggests that

Received March 5, 2004; revised May 20, 2004; accepted May 25, 2004.

This work was supported by the Adler Foundation (R.M.S.), National Institutes of Health Grant 2P01 NS037520-02 (R.M.S.), and Swiss Fonds National de la Recherche Scientifique Grants 31-56930-99 (P.M. and L.P.) and 3100A0-100679 (L.P.). We thank Daniela Kaufer, Dave Schaal, and Sheila Brooke for critical reading of this manuscript and Beth Hoyte for help with the figures.

Correspondence should be addressed to Robert M. Sapolsky, Gilbert Laboratory, Mail Code 5020, Department of Biological Sciences, Stanford University, Stanford, CA 94305-5020. E-mail: sapolsky@stanford.edu.

DOI:10.1523/JNEUROSCI.0805-04.2004

Copyright $\odot 2004$ Society for Neuroscience $\quad 0270-6474 / 04 / 246202-07 \$ 15.00 / 0$ lactate, not glucose, is the major neuronal energy substrate after an insult (Schurr et al., 1997a,b; Cater et al., 2001, 2003). Lactate is produced in glial cells and is delivered to neurons by the glia. This has been termed the astrocyte-neuron lactate shuttle model: astrocytes with endfeet-bordering capillaries take up glucose from the circulation (or the extracellular space), converting it to lactate for export to neurons. Commensurate with this model, monocarboxylate (including lactate) transporters have been found on neurons and astrocytes both in vitro and in vivo. Neurons primarily express the monocarboxylate transporter MCT2 (Bergersen et al., 2001; Pierre et al., 2002; Debernardi et al., 2003), the kinetics of which favor lactate uptake, whereas astrocytes express either MCT1 or MCT4 (Pierre et al., 2000; Bergersen et al., 2001; Debernardi et al., 2003), the kinetics of which favor lactate release, which is consistent with the lactate shuttle model. This lactate shuttle is stimulated during excitotoxic insults by increased concentrations of glutamate in the extracellular space. Moreover, changes in lactate concentrations occur with no change in oxygen content, indicating that this is "intentional" lactate generation, rather than merely secondary to anaerobic metabolism (Hu and Wilson, 1997). Neurons not only use such lactate but recover more effectively from excitotoxic insults when using lactate rather than glucose alone (Schurr et al., 1997a,b; Cater et al., 2001, 2003). This is probably because fewer biochemical steps are required for oxidative phosphorylation from lactate than from glucose, and glucose, unlike lactate, requires an initial investment of ATP to yield energy.

This changing picture of cerebral metabolism suggests that although overexpression of glucose transporters in neurons may be neuroprotective, it should be particularly protective to overexpress either glucose transporters in astrocytes (thereby increasing the availability of glucose for conversion to lactate) or a lactate transporter in neurons (to increase lactate utilization). In this 
paper, we report the neuroprotective potential of each of these strategies and, with dual-gene, dual-cell type therapy, show the synergistic effect of combining the two approaches.

\section{Materials and Methods \\ Cell culture}

Mixed cultures. Primary mixed hippocampal cultures were grown from embryonic day (E) 18 Sprague Dawley rats (Simonsen Laboratories, Gilroy, CA) by standard published methods (Brooke et al., 1997). These cultures are typically $20-30 \%$ neurons and $70-80 \%$ glia.

Neuron-enriched cultures. On day 3, mixed hippocampal cultures were treated with cytosine arabinoside (Sigma, St. Louis, MO) at a final concentration of $10 \mu \mathrm{M}$. When these cells were used on days 10-12, they consisted of 70-90\% neurons (Banker and Goslin, 1991).

Glia-enriched cultures. Glial cultures were made by a standard method, which yields $90-100 \%$ glia. In brief, mixed cortical cultures from E18 rats were grown in poly-D-lysine flasks for $5 \mathrm{~d}$ and then trypsinized and plated into 48-well plates or inserts (Transwell, $3.0 \mu \mathrm{M}$ pore size; Corning, Corning, NY) and grown in MEM (Invitrogen, Grand Island, NY) supplemented with $50 \mathrm{~mm}$ (final concentration) glucose, $5 \%$ horse serum, $0.25 \mu \mathrm{g} / \mathrm{ml}$ fungazone, and penicillin/streptomycin. Cells were used 4-7 $\mathrm{d}$ later when they reached $60-100 \%$ confluency.

\section{Generation of amplicon plasmids}

Generation of the GLUT1 amplicon has been described previously (Lawrence et al., 1996). The DNA coding for the rat MCT2 gene was cloned into the bicistronic expression plasmid p $\alpha 4 \alpha 22 \beta$ gal. The viral DNA and E. coli $\beta$-galactosidase ( $\beta$-gal) reporter gene were placed under the control of the herpes simplex virus (HSV) $\alpha 4$ and $\alpha 22$ immediate early promoters, respectively (see Fig. 1). The control plasmid, p $\alpha 4 \mathrm{~s} \beta$ gal, is a monocistronic plasmid containing only the $\beta$-galactosidase gene (see Fig. 1).

\section{Generation of viral vectors}

Viral vectors were produced as described previously (Ho, 1994). Transient transfection of plasmids into E5 cells (African green monkey Vero cells stably transfected with the HSV $\alpha 4$ gene required for propagation) was followed $24 \mathrm{hr}$ later by superinfection with the helper virus d120, a defective virus required for the replication and packaging of the amplicon. Once complete cytopathic effect was observed, the supernatant was collected, sonicated, and purified on a $25 \%$ sucrose gradient.

\section{Viral infection of neuronal and glial cultures}

The various cell cultures were infected with the indicated vectors at a concentration of 5.5-7.5 $\times 10^{4}$ virus particles per well, which is approximately equivalent to a multiplicity of infection of 0.3-0.5. All subsequent assays were done 18-24 hr after infection. The actual titers of the viruses are given below in units of virus particles per milliliter; parentheses indicate vector virus to helper virus ratio. In all figures, the experiments were repeated several times, and in many cases, different batches of virus were used in the repeated experiments; the different virus preparations used within each experiment were matched as best as possible, so they had a similar titer and vector:helper ratio to control for any effects of the helper virus or cytotoxicity. However, it was not possible to match viral titers and ratios in Figure 5, in which there was excessive total virus added in the GLUT1 wells. Therefore, in one experiment, we examined one of the endpoints seemingly altered by GLUT1 overexpression (we arbitrarily chose lactate accumulation), testing whether that effect was instead caused by the helper virus. We did this by augmenting the amount of control vector plus helper virus up to the level of GLUT1 vector plus helper virus. This had very little effect on lactate accumulation, suggesting that variation in helper virus over an approximate order of magnitude had no effect on the parameter measured. Figure 2: control, $1.9 \times 10^{7}(1: 3.6) ; \mathrm{MCT} 2,1.3 \times 10^{7}(1: 2.9)$. Figures 3 and 4: control, $3.5 \times 10^{7}(1: 1) ;$ MCT2, $2.9 \times 10^{7}(1: 1)$; control, $5.4 \times 10^{6}(1: 2.5)$; MCT2, $5.1 \times 10^{6}(1: 1.8)$; control, $1.9 \times 10^{7}(1: 1.3)$; MCT2, $1.9 \times 10^{7}$ (1:1.6). Figure $5 a$ : control, $3.3 \times 10^{7}(1: 1.2)$; GLUT1, $9.7 \times 10^{6}(1: 12.4)$; control, $1 \times 10^{7}(1: 3.7)$; GLUT1, $1.3 \times 10^{7}(1: 3)$. Figure $5 b$ : control, $3.3 \times 10^{7}(1: 1.2)$; GLUT1, $9.7 \times 10^{6}(1: 12.4)$. Figure 7: control, $7.4 \times 10^{6}$ (1:3); GLUT1, $7.3 \times 10^{6}(1: 2.4) ;$ MCT2, $4.6 \times 10^{7}(1: 1.4)$.

\section{MCT2 immunostaining and image analysis}

Neuron-enriched cultures were fixed with ice-cold methanol and blocked in 5\% milk/PBS (30 min, room temperature). The cells were sequentially labeled for $\beta$-gal (to identify infected cells) using anti- $\beta$-gal $(1: 2000 ; 1 \mathrm{hr}$, room temperature; Sigma) detected with Texas Redconjugated anti-mouse Igs ( $1: 300 ; 0.5 \mathrm{hr}$, room temperature; Vector Laboratories, Burlingame, CA) followed by anti-MCT2 (Pierre et al., 2000) $\left(1: 250 ; 18-20 \mathrm{hr} ; 4^{\circ} \mathrm{C}\right)$ detected with FITC-conjugated anti-rabbit Ig ( $1: 300 ; 1 \mathrm{hr}$ room temperature; Vector Laboratories). Finally, $4^{\prime}, 6^{\prime}-$ diamidino-2-phenylindole (DAPI) $(1: 1000 ; 10 \mathrm{~min}$, room temperature; Sigma) was added to stain cell nuclei. The cells were examined with a fluorescence microscope, and images were captured using a Hamamatsu (Bridgewater, NJ) camera. Ten fields were imaged per well. The intensity of the MCT2 signal per cell per field was determined using the MetaMorph software (Universal Imaging, West Chester, PA). In brief, a uniform-sized circular template was placed over the cell body of all cells in the MCT2 image, and the average integrated pixel intensity per template was measured. From this measurement, the average MCT2 signal of infected cells (as determined by $\beta$-gal expression) per well was calculated and expressed as a percentage of the average MCT2 signal of uninfected cells in the same well.

\section{Uptake assays}

These were done as described previously (Bliss and Sapolsky, 2001).

Glucose uptake. Cultures were incubated for $3 \mathrm{~min}$ at $37^{\circ} \mathrm{C}$ in bicarbonate buffer containing $\left[{ }^{14} \mathrm{C}\right]$ deoxyglucose $(2-3 \mu \mathrm{Ci} / \mathrm{ml}$, equivalent to 6.5-9.5 $\mu \mathrm{m}$; PerkinElmer Life Sciences, Boston, NY) and $5 \mathrm{~mm}$ glucose. The reaction was stopped with phloretin, and the cells were lysed with $1 \%$ (v/v) Triton X-100. The lysate was assayed for ${ }^{14} \mathrm{C}$ radioactivity by liquid scintillation counting, and counts were normalized for protein (measured using the BCA protein assay kit; Pierce, Rockford, IL).

Lactate utilization. Cultures were treated with the indicated concentrations of lactate and glutamate for $6 \mathrm{hr}$ and then incubated for $3 \mathrm{~min}$ at $37^{\circ} \mathrm{C}$ in Krebs Ringer phosphate HEPES (KRPH) buffer containing $\left[{ }^{14} \mathrm{C}\right]$ lactic acid ( $1 \mu \mathrm{Ci} / \mathrm{ml}$, equivalent to $7 \mu \mathrm{m}$; PerkinElmer Life Sciences). The reaction was stopped by aspirating off the transport buffer and washing twice in ice-cold KRPH buffer. Lysates were assayed as above.

Lactate accumulation assay. Cultures were incubated in bicarbonate buffer containing $5 \mathrm{~mm}$ glucose $\pm 500 \mu \mathrm{M}$ glutamate for $30 \mathrm{~min}$ at $37^{\circ} \mathrm{C}$. The lactate that accumulated in the buffer during this time was measured by the standard nicotinamide adenine dinucleotide spectrophotometric assay (Bliss and Sapolsky, 2001) and normalized for protein (measured using the BCA protein assay kit; Pierce).

\section{Neurological insults and quantification of cell survival}

Glutamate and sodium cyanide toxicity in mixed cultures. Mixed hippocampal cultures were spiked with the indicated final concentrations of lactate and either $50 \mu \mathrm{m}$ glutamate or $5 \mathrm{~mm}$ sodium cyanide. Twelve hours postinsult, cells were fixed with $0.5 \%$ gluteraldehyde and stained with X-Gal solution (5-bromo-4-chloro-3-indolyl-b-D-galactopyranoside; Molecular Probes, Eugene, OR) [0.05\% (w/v) X-Gal, 0.5\% (v/v) DMSO, $12.5 \mathrm{~mm}$ ferric cyanide, $12.5 \mathrm{~mm}$ ferrous cyanide, $1.65 \mathrm{~mm} \mathrm{MgCl}$ in PBS] to show infected cells. Cells were visualized by light microscopy, and the number of healthy infected cells (as defined by having several well-formed neurites) was counted.

Glutamate toxicity in laminar cultures. Approximately $19 \mathrm{hr}$ after infection, inserts containing the virus-infected glia were placed in wells containing neuronal-enriched cultures to form a laminar culture. The media in the inserts were supplemented with glucose ( $15 \mathrm{~mm}$ final glucose concentration), and the laminar cultures were incubated for $1 \mathrm{hr}$ at $37^{\circ} \mathrm{C}$ to allow equilibration between the media in the inserts and that in the wells. Glutamate $(5-20 \mu \mathrm{M}$; predetermined weekly to give $\sim 50 \%$ killing of neurons in the absence of vector) was spiked into the media, and $7 \mathrm{hr}$ postinsult neuronal survival was determined using the 2,3'-azinobis(ethylbenzothiazoline-6-sulfonic) acid (ABTS) assay (Brooke et al., 1999). In brief, cells were immunostained with a MAP2 (neuronal marker) antibody (Sigma), a secondary antibody, followed by Vectastain $\mathrm{ABC}$ and ABTS kits (all from Vector Laboratories). ABTS produces a green-colored product that can be read in an ELISA reader to give a measure of the number of neurons remaining in the culture. 


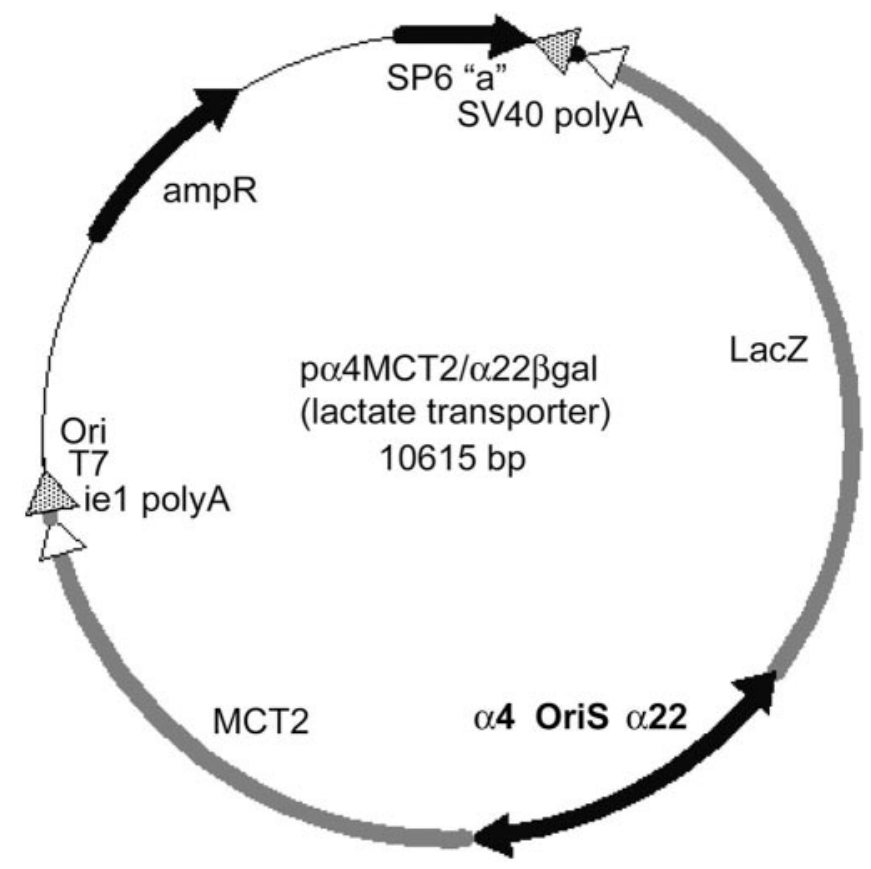

Figure 1. Schematic diagram showing the structure of the amplicon $p \alpha 4 \mathrm{mct} 2 / \alpha 22 \beta \mathrm{gal}$. The $m c t 2$ and lacZ transcriptional units are driven by the HSV $\alpha 4$ and $\alpha 22$ promoters, respectively, and terminated by ie1-polyA and SV40 polyA signals, respectively. The oris and $a$ sequences from HSV-1 provide the necessary replication signals. The control amplicon, $\mathrm{p} \alpha 22 \beta \mathrm{gal}$, is similar to $\mathrm{p} \alpha 4 \mathrm{mct} 2 / \alpha 22 \beta \mathrm{gal}$, except the $m c t 2$ and ie 1 polyA sequences are not included.

Statistical analysis. Data are expressed as a mean \pm SEM. Statistical analysis was performed using Student's $t$ test, one-way or a two-way ANOVA followed by a Tukey's post hoc test, as indicated. Data are taken from at least three separate experiments, except Figures $2 B$ and $5 A$, which were from two separate experiments.

\section{Results}

Monocarboxylate transporter overexpression in neurons

To test the hypothesis that increasing lactate availability to neurons is neuroprotective, we first used a single-cell, single-gene strategy. We constructed a herpes simplex virus amplicon containing the rat neuronal monocarboxylate transporter gene MCT2, which transports lactate (Fig. 1). Cells in neuronenriched hippocampal cultures infected with the MCT2 vector (as shown by expression of the reporter gene $\beta$-gal) showed significantly higher MCT2 staining intensity compared with cultures infected with control vector (Fig. 2). It should be noted that MCT2 expression was significantly upregulated in cells infected with either vector relative to uninfected cells (Fig. $2 B$ ).

To determine whether the increase in number of monocarboxylate transporters was functionally significant, we measured lactate uptake and utilization. In the absence of an insult, there was no significant difference between basal lactate utilization in control-infected or MCT2-infected mixed cultures (Fig. 3), indicating that in the absence of an energy demand, monocarboxylate transporters are not a limiting factor for lactate utilization. In contrast, exposure to $100 \mu \mathrm{M}$ glutamate (an $\mathrm{LC}_{50}$ dose) increased lactate utilization $28 \%(p<0.001)$ in cells overexpressing MCT2 compared with control-infected cells (Fig. 3).

MCT2 overexpression also significantly decreased the neurotoxicity of glutamate (Fig. 4). Increasing lactate concentration within a physiological range (Demestre et al., 1997; Schurr and Rigor, 1998; Shram et al., 1998) resulted in enhanced protection, reaching $100 \%$ protection at $10 \mathrm{~mm}$ lactate (Fig. 4). In contrast, MCT2 overexpression did not protect against the neurotoxicity caused by the metabolic inhibitor cyanide over a range $(0-10$ $\mathrm{mm}$ ) of lactate concentrations (data not shown). Thus, monocarboxylate transporter overexpression is neuroprotective specifically against an excitotoxic insult.

\section{Glucose transporter overexpression in astrocytes}

According to the astrocyte-neuron lactate shuttle model, another way to increase lactate availability to neurons is to increase the amount of glucose taken up by astrocytes; this should enhance the conversion of glucose to lactate and thus enhance glial lactate release. Therefore, we overexpressed GLUT1 in cortical glial cultures. This led to a significant increase in glucose uptake (Fig. $5 A$ ) and enhanced extracellular accumulation of lactate (Fig. $5 B$ ) independent of glutamate status (two-way ANOVA; $p<0$.001; Tukey post hoc test). This effect was more pronounced in the presence of glutamate ( $p<0.05$; Tukey post hoc test) (Fig. $5 B$ ). Commensurate with other studies (Pellerin and Magistretti, 1994), glutamate alone, independent of the vector, also significantly increased lactate accumulation in the media (two-way ANOVA; $p<0.001$; Tukey post hoc test) (Fig. 5B).

Given our data showing that lactate is neuroprotective, we next determined whether GLUT1 overexpression in glia could protect neighboring neurons from glutamate. Because HSV is neurotropic, preferentially infecting neurons over glia in a mixed culture and only significantly infecting glia in the absence of neurons (Fink et al., 2000), pure glial cultures were infected with GLUT1 or control vector before being combined with neuronal cultures in a laminar culture system (Fig. 6a). Glutamate toxicity was reduced in neurons in the presence of glia overexpressing GLUT1 relative to neurons in the presence of control vectorinfected glia (Fig. 6b). Thus, overexpression of GLUT1 in glial cells indirectly decreased excitotoxic neurotoxicity.

\section{Combined gene therapy with GLUT1 and MCT2}

We next used the laminar culture system again to determine whether overexpression of both GLUT1 in glia and MCT2 in neurons would be more protective than either alone. Before combining the two cultures, neurons were infected with either control or MCT2 vector, and glia were infected with either control or GLUT1 vector (Fig. 7). When both glia and neurons were infected with control vector, glutamate caused $\sim 50 \%$ toxicity. Single-gene therapy [i.e., either control/GLUT1 or MCT2/control (neuronal vector/glial vector)] showed a strong trend toward being protective under these specific experimental conditions $(p<0.10$, comparing either with control/control; Tukey's test following one-way ANOVA). The dual-gene approach (MCT2 in neurons and GLUT1 in glia) was significantly protective $(p<$ 0.05; compared with control/control; Tukey's post hoc test), providing a higher level of neuronal survival than either single-gene condition. Quite unexpectedly, neuronal survival in the MCT2/ GLUT1 cultures in the presence of glutamate was considerably $>100 \%$ compared with MCT2/GLUT1 cultures in the absence of glutamate.

\section{Discussion}

As its two key points, this paper: (1) adds support to the emerging picture of the importance of lactate utilization during excitotoxic insults, and (2) demonstrates the feasibility of a novel dual-cell, dual-gene therapy approach. Before considering each, we will first consider a technical issue concerning the study and the paradox in Figure 7 that glutamate appears to be neuroprotective. 


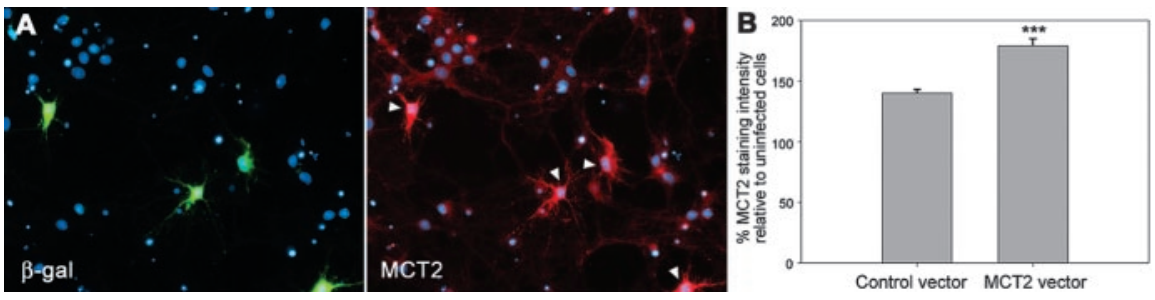

Figure 2. Infection with the lactate transporter (MCT2) vector increases MCT2 protein expression. $a$, Fluorescent images of neuron-enriched cultures infected with the MCT2 vector. Infected cells, shown by the expression of the reporter gene (green, arrowheads), show greater MCT2 staining than uninfected cells. Green, $\beta$-gal staining showing infected cells; red, MCT2 staining; blue, DAPI, nuclear stain. $b$, Quantification of the intensity of the MCT2 fluorescent signal. Data are expressed as percentage MCT2 staining in infected cells relative to the intensity of MCT2 staining in uninfected cells, which is set to $100 \%$. ${ }^{* *} p<0.001$, by Student's $t$ test; $n=6$

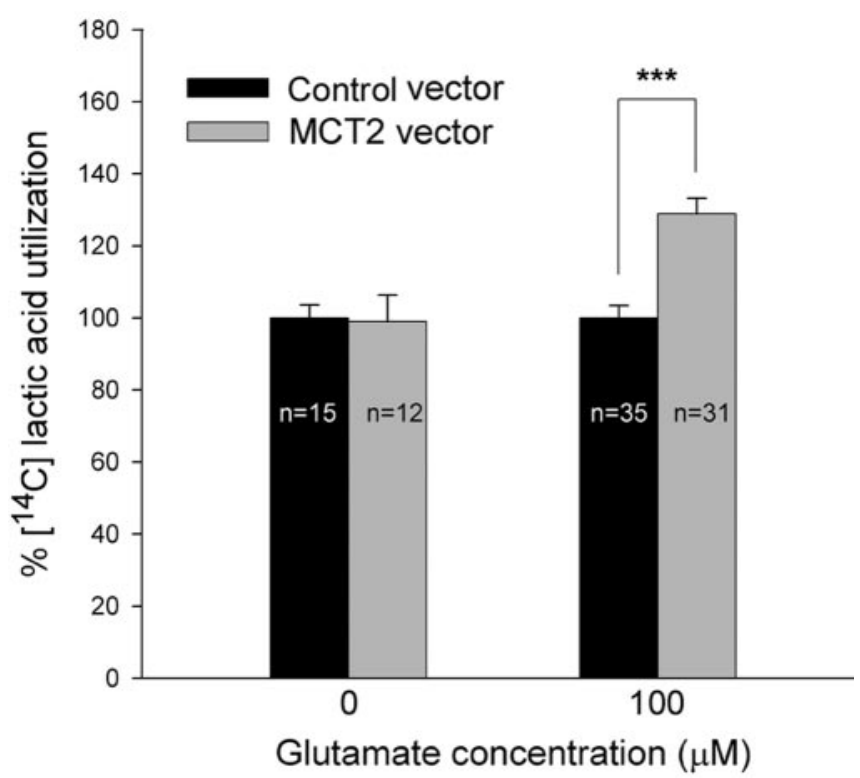

Figure 3. Infection with MCT2 vector increases lactate utilization in the presence of glutamate and $5 \mathrm{~mm}$ lactate. Data are expressed as a percentage of lactate utilization in the controlinfected cultures at the same glutamate concentration. Absolute values for lactate utilization with the control vector in the absence and presence of glutamate are $7.3 \pm 0.3$ and $6.1 \pm 0.5$ $\mathrm{nmol} / \mathrm{mg} / \mathrm{min}$, respectively. ${ }^{* * *} p<0.001$, by Student's $t$ test.

This study and others have shown that glutamate enhances neuronal energetics in the presence of astrocytes, insofar as it increases glial lactate release (Pellerin and Magistretti, 1994) and neuronal lactate utilization (Schurr et al., 1999). This is relevant to the seeming paradox of Figure 7 where, in the dual-gene therapy case, survival after glutamate exposure was significantly higher than in the absence of glutamate. In this enriched neuronal culture system, there is considerable neurotoxicity in the "no glutamate" condition because of viral infection per se and the frequent media changes required (Ho et al., 1995b). Adding glutamate activates glial lactate release and neuronal lactate uptake, thereby increasing energy availability to neurons, protecting them from both the glutamate and viral/media change insult and thereby enhancing neuronal survival. In other words, in this experimental paradigm, the glutamate-induced increase in neuronal energetics outweighs the neurotoxic effects of glutamate.

\section{The lactate/glucose shuttle}

As reviewed, increasing evidence suggests that lactate is a significant energy substrate for neurons. Lactate can be used as an oxi- dative substrate in brain slices (Ide et al., 1969; Fernandez and Medina, 1986), cultured telencephalic neurons (Tabernero et al., 1996; McKenna et al., 2001), aggregated neuronal cultures (Honegger et al., 2002), sympathetic ganglia (Larrabee, 1996), and synaptic terminals (McKenna et al., 1993) and can be preferred to glucose (Bouzier-Sore et al., 2003; Itoh et al., 2003; Smith et al., 2003). In addition, metabolic demands increase neuronal ability to use lactate (Schurr et al., 1999). This involves both increased expression of MCT2 (Pierre et al., 2003) and increased trafficking of lactate through the astrocyteneuron lactate shuttle (Pellerin et al., 1998). This emphasis on the importance of lactate is particularly interesting in the context of hypoxic-ischemic insults. A longstanding literature has shown that the lactate generated by anaerobic metabolism during such insults can be damaging via acidotic mechanisms, and that glucose administration can exacerbate this pathway. It has come to be recognized, however, that such acidotic injury is typically restricted to the ischemic core (Tombaugh and Sapolsky, 1993). Furthermore, recent evidence suggests that preischemic hyperglycemic exacerbation of injury in vivo can be attributable to glucose-induced elevation of glucocorticoid levels and that this, rather than an increase in lactic acidosis, is responsible for the deleterious effect (Schurr et al., 2001a; Schurr, 2002).

Prompted by this novel view of neuroenergetics, we assessed the neuroprotective potential of gene therapy strategies targeting the lactate shuttle. We first increased lactate availability to neurons by overexpressing monocarboxylate transporters in neurons. Under resting conditions, MCT2 overexpression did not enhance lactate utilization. However, higher concentrations of lactate than those we used are reported to stimulate neuronal lactate utilization (Bouzier-Sore et al., 2003; Itoh et al., 2003). Consistent with previous reports (Schurr et al., 1999), we found that glutamate increased lactate utilization in MCT2-overexpressing neurons but not in control neurons. This suggests that during highenergy demand, MCT2 levels could become rate limiting for lactate utilization, and this can be alleviated by MCT2 overexpression.

Lactate can be neuroprotective in vitro after glucose or oxygen deprivation (Izumi et al., 1997; Schurr et al., 1997a,b; Cater et al., 2001, 2003) or glutamate exposure (Schurr et al., 1999) and in vivo after ischemia (Schurr et al., 2001b) or glutamate exposure (Mendelowitsch et al., 2001; Ros et al., 2001). In agreement, we found that in the presence of increasing extracellular lactate, MCT2 overexpression enhanced neuronal survival after exposure to glutamate. Because glutamate decreases cell energy status (Nicholls and Budd, 1998), the most likely explanation for such neuroprotection would be that MCT2 overexpression facilitates entry of a readily utilizable energy source. Indeed, lactate can support neuronal respiration to the same extent as glucose (Pellerin et al., 1998). Commensurate with this, we found that lactate did not protect against sodium cyanide, a respiratory chain inhibitor.

We next determined whether overexpressing GLUT1 in glia was neuroprotective. Such overexpression enhanced both glucose utilization and lactate release, either because GLUT1 is rate limiting for glucose utilization in astrocytes or because its overexpression alters expression or regulation of other rate-limiting glycolytic enzymes. Furthermore, GLUT1 overexpression en- 


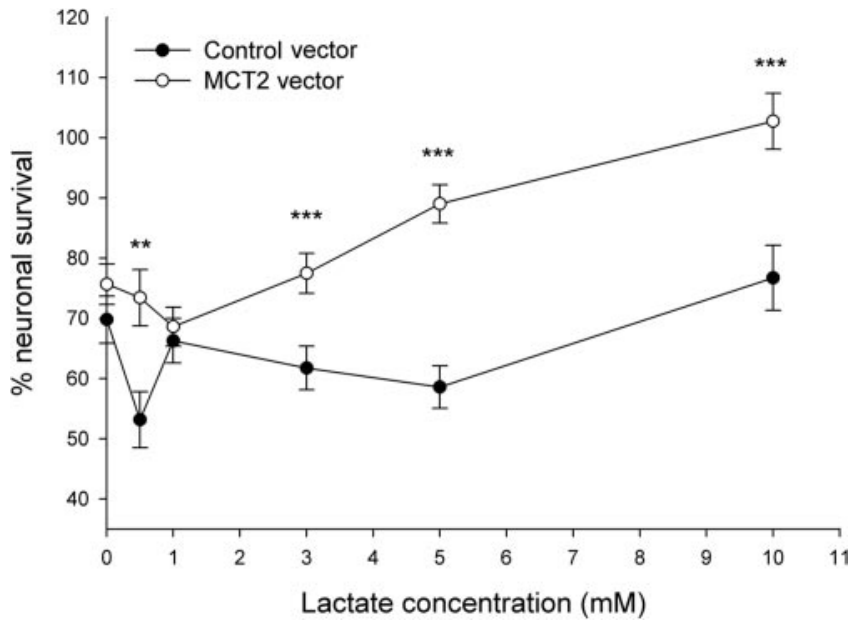

Figure 4. Overexpression of MCT2 protects neurons from an excitotoxic insult. MCT2infected hippocampal cultures show greater neuronal survival after a glutamatergic insult ( 50 $\mu \mathrm{M}$ ) than control-infected cultures ( $p<0.03$, by two-way ANOVA). Increasing lactate concentration in the cell media significantly increases this neuroprotection in MCT2-infected cultures compared with control-infected cultures. Data are expressed as percentage survival of controlinfected cells in the absence of insult at the same lactate concentration; ${ }^{* *} p<0.01$, ${ }^{* *} p<$ 0.001 , compared with control vector at the same lactate concentration, by Tukey post hoc test following two-way ANOVA; $n=12$ for 0.5 and $10 \mathrm{~mm}$ lactate; $n>25$ for all others.
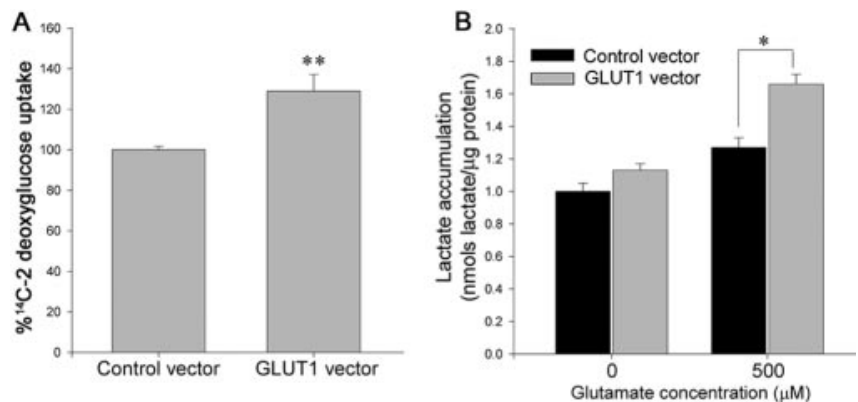

Figure 5. Increasing the number of glucose transporters in glia increases glucose uptake and lactate release. $a$, Glial cells infected with the GLUT1 vector showed enhanced ${ }^{14} \mathrm{C}-2$ deoxyglucose uptake in the presence of $5 \mathrm{~mm}$ glucose compared with cells infected with control vector. Uptake value for the control was $9.3 \pm 1.4 \mathrm{nmol} / \mathrm{mg} / \mathrm{min} ;{ }^{* *} p<0.01$, by Student's $t$ test; $n=$ 8. $b$, Enhanced lactate accumulation in the media. Approximate values of lactate concentration range from $81 \pm 4.5 \mu \mathrm{m}$ (control vector in the absence of glutamate) to $128 \pm 10.3 \mu \mathrm{m}$ (GLUT1 vector in the presence of glutamate); ${ }^{*} p<0.05$, compared with control vector at the same glutamate concentration, by Tukey post hoc test following two-way ANOVA; $n=24$.

hanced glutamate-induced lactate formation in glia. This suggests that GLUT1 overexpression expanded the glial glycolytic capacity, allowing for a more efficient metabolic response during a challenge. Moreover, consistent with the importance of lactate, glutamate was less toxic to neurons in the presence of glia overexpressing GLUT1 than neurons in the presence of glia infected with control vector.

Finally, given that both strategies above were neuroprotective, we tested whether the combination would be more protective than either alone. The experiment was conducted under culture conditions in which overexpression of GLUT1 in glia or MCT2 in neurons alone produced only a trend toward protection. When gene therapy interventions were combined, there was significant protection, thereby confirming that the dual gene therapy is superior to either single gene therapy approach.

As a note of caution, when using an HSV amplicon-helper virus system as we do, the helper virus per se may alter cellular
A

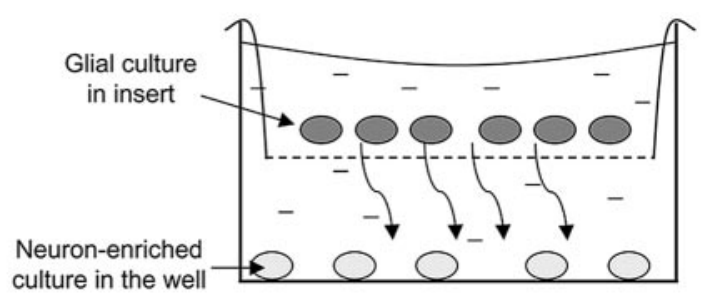

B

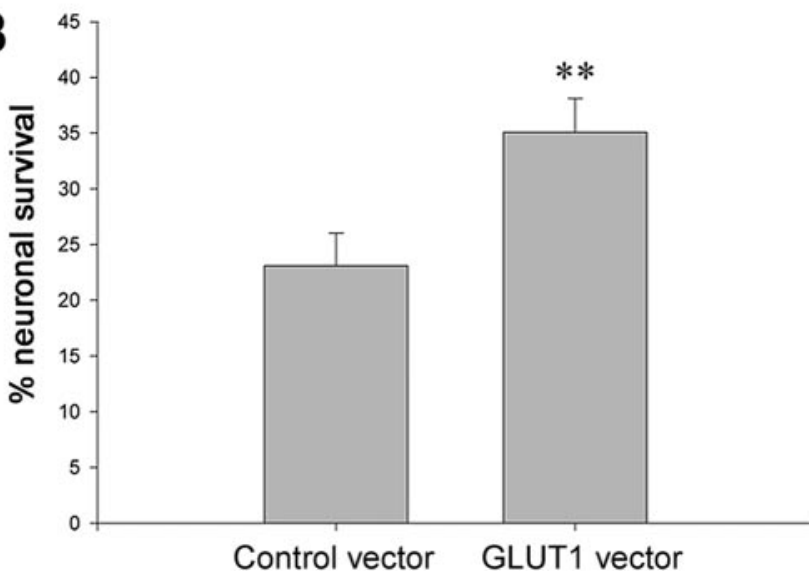

Figure 6. Glia infected with the GLUT1 vector protect neurons from an excitotoxic insult. $a$ Schematic diagram of the laminar culture system. Bent arrows indicate that metabolites released by glia can diffuse to the neurons. $b$, Neurons exposed to glia infected with GLUT1 vector show enhanced survival after a gutamatergic insult $\left(\mathrm{LC}_{50}\right.$ concentration) compared with neurons exposed to glia infected with control vector. Data are expressed as a percentage of neuronal survival in the absence of glutamate under the same vector conditions; ${ }^{* *} p<0.01$, by Student's $t$ test; $n=16$.

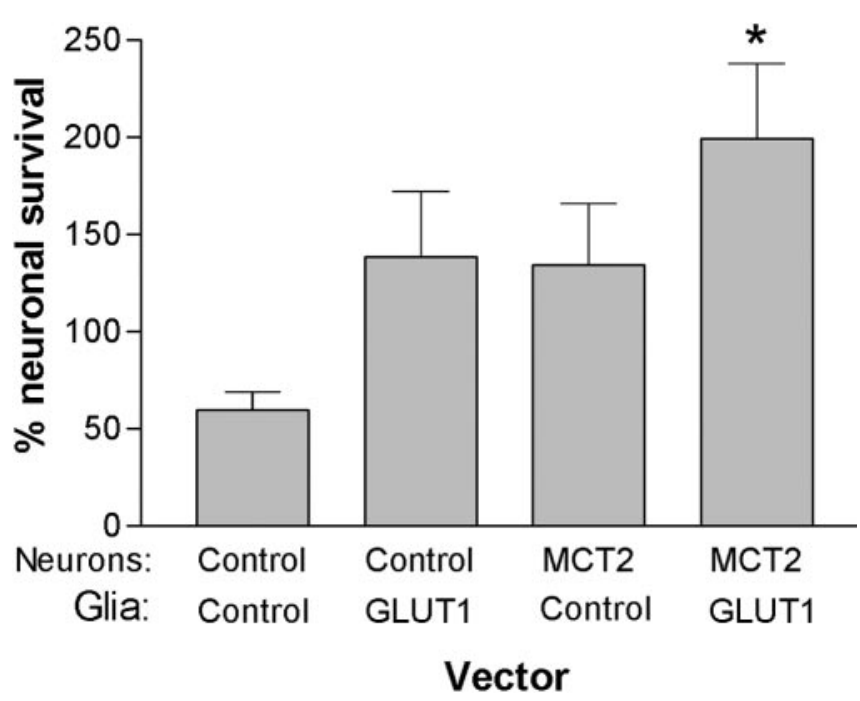

Figure 7. Combined MCT2 and GLUT1 therapy gives greater neuronal protection than either vector alone. Data are expressed as a percentage of survival compared with the same vector combination in the absence of glutamate; ${ }^{*} p<0.05$, compared with the control-control condition, by Tukey post hoc test following one-way ANOVA; $n=14$.

functions and affect the parameters being measured. As detailed in Materials and Methods, we tested for this possibility and found little indication that this was occurring in this case. Thus, although we can't fully exclude the possibility of helper virus effects on our results, we feel that it is unlikely. 


\section{Dual-cell, dual-gene therapy}

Gene therapy in the nervous system has grown increasingly sophisticated, with the development of vectors in which, for example, expression can be cell-type-, pathway-, or region-specific or can be induced by either exogenous or endogenous signals (Sapolsky, 2003). Another advance is the ability to deliver more than a single transgene, an obvious advantage given the functional networks that characterize the biochemistry of the brain. A number of previous gene therapy studies have used vectors that expressed multiple transgenes in the same cell (Krisky et al., 1998; Andsberg et al., 2002; Slimko et al., 2002) or in which the same cells were infected with more than one vector, each expressing a different transgene (Eberhardt et al., 2000; Natsume et al., 2001; Muramatsu et al., 2002). To our knowledge, this is the first report of the overexpression of different transgenes in different cell types to alter the steps in a multicellular pathway.

Extending this dual-cell, dual-gene approach to in vivo studies is plausible but difficult. Given that HSV is strongly neurotropic, intraparenchymal infusion of it is likely to predominantly infect neurons and could be used to deliver MCT2. In contrast, "artificial viruses" made of immunoliposomes are able to deliver transgenes across the blood-brain barrier, and if having glia-specific expression (for example, by the use of a GFAP promoter) would preferentially target astrocytes bordering capillaries (Shi et al., 2001; Zhang et al., 2003), these could be used to deliver GLUT1. Ongoing studies are exploring this possibility.

In conclusion, our data support the astrocyte-neuron lactate shuttle model and the changing picture of cerebral metabolism during excitotoxic neurological insults. Although the use of a dual-cell type, dual-gene approach in a clinical setting is not currently a reality, the present study shows the potential of such an approach and also illustrates how gene therapy studies can reveal basic processes involved in cell-cell interactions.

\section{References}

Andsberg G, Kokaia Z, Klein RL, Muzyczka N, Lindvall O, Mandel RJ (2002) Neuropathological and behavioral consequences of adeno-associated viral vector-mediated continuous intrastriatal neurotrophin delivery in a focal ischemia model in rats. Neurobiol Dis 9:187-204.

Banker G, Goslin K (1991) Culturing nerve cells. Cambridge, MA: MIT.

Bergersen L, Waerhaug O, Helm J, Thomas M, Laake P, Davies AJ, Wilson MC, Halestrap AP, Ottersen OP (2001) A novel postsynaptic density protein: the monocarboxylate transporter MCT2 is co-localized with delta-glutamate receptors in postsynaptic densities of parallel fiberPurkinje cell synapses. Exp Brain Res 136:523-534.

Bliss TM, Sapolsky RM (2001) Interactions among glucose, lactate and adenosine regulate energy substrate utilization in hippocampal cultures. Brain Res 899:134-141.

Bouzier-Sore AK, Voisin P, Canioni P, Magistretti PJ, Pellerin L (2003) Lactate is a preferential oxidative energy substrate over glucose for neurons in culture. J Cereb Blood Flow Metab 23:1298-1306.

Brooke S, Chan R, Howard S, Sapolsky R (1997) Endocrine modulation of the neurotoxicity of gp120: implications for AIDS-related dementia complex. Proc Natl Acad Sci USA 94:9457-9462.

Brooke SM, Bliss TM, Franklin LR, Sapolsky RM (1999) Quantification of neuron survival in monolayer cultures using an enzyme-linked immunosorbent assay approach, rather than by cell counting. Neurosci Lett 267:21-24.

Cater HL, Benham CD, Sundstrom LE (2001) Neuroprotective role of monocarboxylate transport during glucose deprivation in slice cultures of rat hippocampus. J Physiol (Lond) 531:459-466.

Cater HL, Chandratheva A, Benham CD, Morrison B, Sundstrom LE (2003) Lactate and glucose as energy substrates during, and after, oxygen deprivation in rat hippocampal acute and cultured slices. J Neurochem 87:1381-1390.

Debernardi R, Pierre K, Lengacher S, Magistretti PJ, Pellerin L (2003) Cellspecific expression pattern of monocarboxylate transporters in astrocytes and neurons observed in different mouse brain cortical cell cultures. J Neurosci Res 73:141-155.

Demestre M, Boutelle M, Fillenz M (1997) Stimulated release of lactate in freely moving rats is dependent on the uptake of glutamate. J Physiol (Lond) 499:825-832.

Eberhardt O, Coelln RV, Kugler S, Lindenau J, Rathke-Hartlieb S, Gerhardt E, Haid S, Isenmann S, Gravel C, Srinivasan A, Bahr M, Weller M, Dichgans J, Schulz JB (2000) Protection by synergistic effects of adenovirusmediated X-chromosome-linked inhibitor of apoptosis and glial cell linederived neurotrophic factor gene transfer in the 1-methyl-4-phenyl1,2,3,6-tetrahydropyridine model of Parkinson's disease. J Neurosci 20:9126-9134.

Fernandez E, Medina JM (1986) Lactate utilization by the neonatal rat brain in vitro. Competition with glucose and 3-hydroxybutyrate. Biochem J 234:489-492.

Fink DJ, DeLuca NA, Yamada M, Wolfe DP, Glorioso JC (2000) Design and application of HSV vectors for neuroprotection. Gene Ther 7:115-119.

Gupta A, Ho DY, Brooke S, Franklin L, Roy M, McLaughlin J, Fink SL, Sapolsky RM (2001) Neuroprotective effects of an adenoviral vector expressing the glucose transporter: a detailed description of the mediating cellular events. Brain Res 908:49-57.

Ho DY (1994) Amplicon-based herpes simplex virus vectors. Methods Cell Biol 43:191-210.

Ho DY, Saydam TC, Fink SL, Lawrence MS, Sapolsky RM (1995a) Defective herpes simplex virus vectors expressing the rat brain glucose transporter protect cultured neurons from necrotic insults. J Neurochem 65:842-850.

Ho DY, Fink SL, Lawrence MS, Meier TJ, Saydam TC, Dash R, Sapolsky RM (1995b) Herpes simplex virus vector system: analysis of its in vivo and in vitro cytopathic effects. J Neurosci Methods 57:205-215.

Honegger P, Braissant O, Henry H, Boulat O, Bachmann C, Zurich MG, Pardo B (2002) Alteration of amino acid metabolism in neuronal aggregate cultures exposed to hypoglycaemic conditions. J Neurochem 81:1141-1151.

Hu Y, Wilson GS (1997) A temporary local energy pool coupled to neuronal activity: fluctuations of extracellular lactate levels in rat brain monitored with rapid-response enzyme-based sensor. J Neurochem 69:1484-1490.

Ide T, Steinke J, Cahill Jr GF (1969) Metabolic interactions of glucose, lactate, and beta-hydroxybutyrate in rat brain slices. Am J Physiol 217:784-792.

Itoh Y, Esaki T, Shimoji K, Cook M, Law MJ, Kaufman E, Sokoloff L (2003) Dichloroacetate effects on glucose and lactate oxidation by neurons and astroglia in vitro and on glucose utilization by brain in vivo. Proc Natl Acad Sci USA 100:4879-4884.

Izumi Y, Benz AM, Katsuki H, Zorumski CF (1997) Endogenous monocarboxylates sustain hippocampal synaptic function and morphological integrity during energy deprivation. J Neurosci 17:9448-9457.

Krisky DM, Marconi PC, Oligino TJ, Rouse RJ, Fink DJ, Cohen JB, Watkins SC, Glorioso JC (1998) Development of herpes simplex virus replication-defective multigene vectors for combination gene therapy applications. Gene Ther 5:1517-1530.

Larrabee MG (1996) Partitioning of CO2 production between glucose and lactate in excised sympathetic ganglia, with implications for brain. J Neurochem 67:1726-1734.

Lawrence MS, Ho DY, Dash R, Sapolsky RM (1995) Herpes simplex virus vectors overexpressing the glucose transporter gene protect against seizure-induced neuron loss. Proc Natl Acad Sci USA 92:7247-7251.

Lawrence MS, Ho DY, Sun GH, Steinberg GK, Sapolsky RM (1996) Overexpression of $\mathrm{Bcl}-2$ with herpes simplex virus vectors protects CNS neurons against neurological insults in vitro and in vivo. J Neurosci 16:486-496.

McKenna MC, Tildon JT, Stevenson JH, Boatright R, Huang S (1993) Regulation of energy metabolism in synaptic terminals and cultured rat brain astrocytes: differences revealed using aminooxyacetate. Dev Neurosci 15:320-329.

McKenna MC, Hopkins IB, Carey A (2001) Alpha-cyano-4-hydroxycinnamate decreases both glucose and lactate metabolism in neurons and astrocytes: implications for lactate as an energy substrate for neurons. J Neurosci Res 66:747-754.

McLaughlin J, Roozendaal B, Dumas T, Gupta A, Ajilore O, Hsieh J, Ho D, Lawrence M, McGaugh JL, Sapolsky R (2000) Sparing of neuronal function postseizure with gene therapy. Proc Natl Acad Sci USA [Erratum (2001) 98:1317] 97:12804-12809.

Mendelowitsch A, Ritz MF, Ros J, Langemann H, Gratzl O (2001) 17beta- 
Estradiol reduces cortical lesion size in the glutamate excitotoxicity model by enhancing extracellular lactate: a new neuroprotective pathway. Brain Res 901:230-236.

Muramatsu S, Fujimoto K, Ikeguchi K, Shizuma N, Kawasaki K, Ono F, Shen Y, Wang L, Mizukami H, Kume A, Matsumura M, Nagatsu I, Urano F, Ichinose H, Nagatsu T, Terao K, Nakano I, Ozawa K (2002) Behavioral recovery in a primate model of Parkinson's disease by triple transduction of striatal cells with adeno-associated viral vectors expressing dopaminesynthesizing enzymes. Hum Gene Ther 13:345-354.

Natsume A, Mata M, Goss J, Huang S, Wolfe D, Oligino T, Glorioso J, Fink DJ (2001) Bcl-2 and GDNF delivered by HSV-mediated gene transfer act additively to protect dopaminergic neurons from 6-OHDA-induced degeneration. Exp Neurol 169:231-238.

Nicholls DG, Budd SL (1998) Neuronal excitotoxicity: the role of mitochondria. Biofactors 8:287-299.

Pellerin L, Magistretti PJ (1994) Glutamate uptake into astrocytes stimulates aerobic glycolysis: a mechanism coupling neuronal activity to glucose utilization. Proc Natl Acad Sci USA 91:10625-10629.

Pellerin L, Magistretti PJ (2003) Food for thought: challenging the dogmas. J Cereb Blood Flow Metab 23:1282-1286.

Pellerin L, Pellegri G, Bittar PG, Charnay Y, Bouras C, Martin JL, Stella N, Magistretti PJ (1998) Evidence supporting the existence of an activitydependent astrocyte-neuron lactate shuttle. Dev Neurosci 20:291-299.

Pierre K, Pellerin L, Debernardi R, Riederer BM, Magistretti PJ (2000) Cellspecific localization of monocarboxylate transporters, MCT1 and MCT2, in the adult mouse brain revealed by double immunohistochemical labeling and confocal microscopy. Neuroscience 100:617-627.

Pierre K, Magistretti PJ, Pellerin L (2002) MCT2 is a major neuronal monocarboxylate transporter in the adult mouse brain. J Cereb Blood Flow Metab 22:586-595.

Pierre K, Debernardi R, Magistretti PJ, Pellerin L (2003) Noradrenaline enhances monocarboxylate transporter 2 expression in cultured mouse cortical neurons via a translational regulation. J Neurochem 86:1468-1476.

Ros J, Pecinska N, Alessandri B, Landolt H, Fillenz M (2001) Lactate reduces glutamate-induced neurotoxicity in rat cortex. J Neurosci Res 66:790-794.

Sapolsky RM (2003) Neuroprotective gene therapy against acute neurological insults. Nat Rev Neurosci 4:61-69.

Schurr A (2002) Energy metabolism, stress hormones and neural recovery from cerebral ischemia/hypoxia. Neurochem Int 41:1-8.
Schurr A, Rigor BM (1998) Brain anaerobic lactate production: a suicide note or a survival kit? Dev Neurosci 20:348-357.

Schurr A, Payne RS, Miller JJ, Rigor BM (1997a) Brain lactate is an obligatory aerobic energy substrate for functional recovery after hypoxia: further in vitro validation. J Neurochem 69:423-426.

Schurr A, Payne RS, Miller JJ, Rigor BM (1997b) Brain lactate, not glucose, fuels the recovery of synaptic function from hypoxia upon reoxygenation: an in vitro study. Brain Res 744:105-111.

Schurr A, Miller JJ, Payne RS, Rigor BM (1999) An increase in lactate output by brain tissue serves to meet the energy needs of glutamate-activated neurons. J Neurosci 19:34-39.

Schurr A, Payne RS, Miller JJ, Tseng MT (2001a) Preischemic hyperglycemia-aggravated damage: evidence that lactate utilization is beneficial and glucose-induced corticosterone release is detrimental. J Neurosci Res 66:782-789.

Schurr A, Payne RS, Miller JJ, Tseng MT, Rigor BM (2001b) Blockade of lactate transport exacerbates delayed neuronal damage in a rat model of cerebral ischemia. Brain Res 895:268-272.

Shi N, Zhang Y, Zhu C, Boado RJ, Pardridge WM (2001) Brain-specific expression of an exogenous gene after i.v. administration. Proc Natl Acad Sci USA 98:12754-12759.

Shram NF, Netchiporouk LI, Martelet C, Jaffrezic-Renault N, Bonnet C, Cespuglio R (1998) In vivo voltammetric detection of rat brain lactate with carbon fiber microelectrodes coated with lactate oxidase. Anal Chem 70:2618-2622.

Slimko EM, McKinney S, Anderson DJ, Davidson N, Lester HA (2002) Selective electrical silencing of mammalian neurons in vitro by the use of invertebrate ligand-gated chloride channels. J Neurosci 22: 7373-7379.

Smith D, Pernet A, Hallett WA, Bingham E, Marsden PK, Amiel SA (2003) Lactate: a preferred fuel for human brain metabolism in vivo. J Cereb Blood Flow Metab 23:658-664.

Tabernero A, Vicario C, Medina JM (1996) Lactate spares glucose as a metabolic fuel in neurons and astrocytes from primary culture. Neurosci Res 26:369-376.

Tombaugh GC, Sapolsky RM (1993) Evolving concepts about the role of acidosis in ischemic neuropathology. J Neurochem 61:793-803.

Zhang Y, Schlachetzki F, Pardridge WM (2003) Global non-viral gene transfer to the primate brain following intravenous administration. Mol Ther 7:11-18. 